\title{
Schwangerschaftsprävention bei jungen Patientinnen
}

\author{
S. Anthuber, München $\quad H$. Hepp, München $\quad$ D. Fink, Zürich $\quad R$. Kimmig, Essen \\ U. Lang, Graz
}

\section{Zusammenfassung}

Stetig steigende Schwangerschafts- und Schwangerschaftsabbruchszahlen bei Teenagern erfordern eine gute Aufklärung, Kenntnisse über normale Entwicklungsvorgänge und das Verhütungsverhalten von Jugendlichen. Moderne, complianceunabhängige und nebenwirkungsarme Kontrazeptiva sollten verordnet werden und die Rechtsgrundlage dazu bekannt sein. $\mathrm{Ob}$ eine geeignete Kontrazeption für den Mann auch bei Jugendlichen anwendbar ist, ist Gegenstand von Diskussionen. Die Beratung körperlich und geistig behinderter Jugendlicher stellt besondere Anforderungen an den Frauenarzt und die Frauenärztin. Jugendliche sind anfälliger für HPV-Infektionen und müssen dahingehend untersucht, beraten und auf die Assoziation mit einer Zervixdysplasie hingewiesen werden.

\section{Contraception in Young Adolescents}

The continuing increase in teenage pregnancies and abortions calls for more effective sex education on the one hand, including a detailed explication of the (perfectly normal) changes an adolescent body is undergoing, but we also need to understand the way teenagers actually do use contraceptives. Modern contraception for teenagers should not cause any side effects and its efficacy must not be linked to compliance. Gynaecologists must acquaint themselves with the laws covering the prescription of contraceptives to adolescents. Whether hormonal contraceptives for men are also suitable for teenage boys remains to be seen. Advising mentally or physically handicapped teenagers or those with chronic diseases on their contraceptive options is particularly challenging. Adolescents are more prone to contract HPV infections and thus need to be screened, counselled and told about the association between HPV and cervical dysplasia.

Copyright (C 2005 S. Karger AG, Basel
Noch nie hatten Teenager einen leichteren und breiteren Zugang zu Informationen und Medien. Auch ihre Eltern sind bereits mit der durch die «Pille» bedingten Trennung von sexuellem Erleben und Fortpflanzung aufgewachsen. Und dennoch: noch nie gab es so viele registrierte Teenagerschwangerschaften wie heute. Auch die Anzahl der Schwangerschaftsabbrüche bei Minderjährigen steigt nach wie vor stetig an, insbesondere der prozentuale Anteil der unter 15-Jährigen. Frau Gisela Gille, die Vorsitzende der Ärztlichen Gesellschaft zur Gesundheitsförderung der Frau, nennt in ihrem Beitrag folgende Gründe: Eine mangelhafte, nicht an den Bedürfnissen der Mädchen orientierte Aufklärung, die Sexualisierung durch die Medien, die mangelnden Perspektiven für die Mädchen am Arbeitsmarkt, zu früh oder zu intensiv geweckte Beziehungssehnsüchte und die bewusste Flucht durch das Herbeiführen einer Schwangerschaft in eine vermeintlich «heile Welt». Dabei gibt es eine offensichtliche Diskrepanz zwischen dem immer früher eintretenden körperlichen und psychosexuellen Reifungsprozess. Typisch für noch unreife Mädchen ist ein geringes Selbstwertgefühl, ein hohes Neugierverhalten, eine grosse Risikobereitschaft mit der mangelnden Fähigkeit zu vorausschauendem Handeln. Dabei treffen sie dann auf die stark libidinös geprägten Jungen. Befragt man Jugendliche, so sind sie aber nur sehr vage mit den körperlichen Veränderungen und physiologischen Grundlagen, die zur Fortpflanzungsfähigkeit führen, vertraut. Weder der Zeitpunkt der Empfängnisbereitschaft noch die allgemeinen physiologischen Abläufe des Körpers sind ihnen

\section{KARGER \\ Fax +4161306 1234 \\ E-Mail karger@karger.ch}

www.karger.com
(C) 2005 S. Karger AG, Basel

www.karger.com/ggr
Prof. Dr. H. Hepp

Ehemaliger Direktor der Klinik und Poliklinik für Frauenheilkunde und Geburtshilfe im Klinikum Grosshadern der Ludwig-Maximilian-Universität, Schlossstrasse 15 DE-82266 Buch a. Ammersee (Deutschland)

E-Mail hermann_hepp@gmx.de 
bekannt. Aus Unwissenheit und erkennbar nicht bedarfsgerechter Aufklärung resultiert dann auch eine unzureichende Körperidentität und -akzeptanz. So wird z.B. die Menstruation nicht als Ausdruck von Weiblichkeit und einem intakten endokrinen Zyklus, sondern als störend empfunden.

Es ist die wichtige Aufgabe der Frauenärzte und -ärztinnen, die Jugendlichen in einer jugendgerechten Atmosphäre aufzuklären, behutsam auf ihre Bedürfnisse einzugehen und damit ihr Selbstwertgefühl zu stärken. Besonders wichtig sind Kenntnisse über moderne, complianceunabhängige und nebenwirkungsarme Kontrazeptiva. Dabei ist es von grosser Bedeutung, die Ausführungen der AG Medizinrecht zur Verordnung von Kontrazeptiva an Minderjährige zu kennen und die derzeit gültige Rechtssprechung zu beachten.

Orale Kontrazeptiva sind die am häufigsten verordneten Verhütungsmittel an Jugendliche. Sie wirken reversibel, sind gut verträglich, sicher und haben je nach Gestagenzusatz zusätzliche positive Nebeneffekte. Im Langzyklus wirken monophasische orale Kontrazeptiva stärker kontrazeptiv und zusätzlich positiv auf Dysmenorrhöe, PCOS, Pillenpausenmigräne und andere zyklusassoziierte Beschwerden. Aber auch Langzeitkontrazeptiva, wie subdermal zu implantierende Stäbchen oder das hormonhaltige Intrauterinpessar, sind mittlerweile etabliert. Neuere hormonhaltige Verhütungsmittel sind Vaginalring und Verhütungspflaster.
Kontrazeptive Methoden für den Mann waren bislang entweder weniger effektiv (z.B. das Kondom) oder nicht reversibel. Ein hormonhaltiges Kontrazeptivum für den Mann muss sicher, reversibel und praktikabel in der Anwendung sein. Axel Kamischke beschreibt den derzeitigen Entwicklungstand hierzu. Ob es eine Alternative für Jugendliche darstellt, muss aber noch geklärt werden.

Auch die Kontrazeptionsberatung geistig und körperlich behinderter oder chronisch kranker Jugendlicher erfordert ein hohes Mass an Kompetenz, Geduld und Einfühlungsvermögen. Nach Francesca Navratil aus Zürich mangelt es gerade bei diesen Jugendlichen an einer korrekten Aufklärung, obgleich sich ihre sexuellen Bedürfnisse nicht von denen anderer Jugendlicher unterscheiden. Eine individuelle Beratung ist hier ganz besonders wichtig, da neben den Zielen Schwangerschaftsverhütung und selbst bestimmte Sexualität vor allem auch die Prävention sexueller Gewalt hinzukommt.

Die Entstehung des Zervixkarzinoms ist nahezu hundertprozentig mit der HPV-Infektion assoziiert. Jugendliche sind offensichtlich begünstigt sich in jungen Jahren mit HPV zu infizieren. Es gibt Hinweise darauf, dass die sekundäre Immunantwort der Jugendlichen auf die Infektion weniger effizient ist als in höheren Altersgruppen. Dies wird in dem Beitrag von Olaf Reich erläutet, der auch der Frage nachgeht, ob die frühe Kohabitarche eine Risikosteigerung bedeutet. 